\title{
About Spatial Rigid Memory CHARACTERISTICS
}

\author{
Comanescu, A.; Comanescu, D.; \\ FilipoiU, I. D. \& Alionte, C.G.
}

Abstract: The paper presents how the computer aided synthesis of spatial cam can be done based on kinematical elements for various mechanisms applied in industrial equipments. The method uses the follower planar translation displacement wich can be described by different motion laws. Thus, the spatial cams profile parameters are determined for various laws of the follower and by using proper design software the profile parameters are used to draw the cylindrical, conical and hyperbolical cams and their profiles.

Key words: CAD, cams, analisys, synthesis
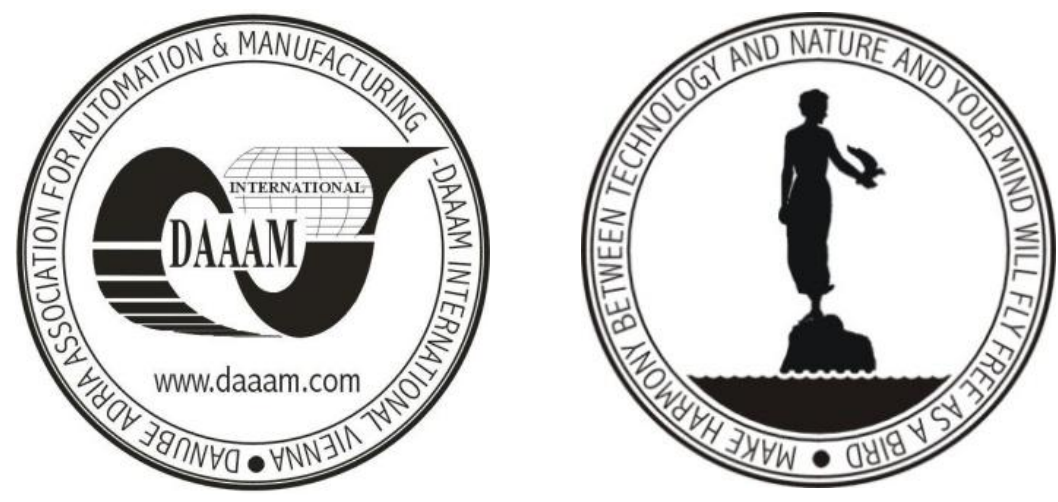

Authors' data: Univ.Prof. Comanescu, A[driana]; Assoc. Prof. Comanescu D[inu]; Assoc. Prof. Filipoiu I[oan] D[an]; Alionte C[ristian] G[abriel], University Politehnica of Bucharest, Splaiul Independentei 313, 060042, Bucharest, Romania, adrianacomanescu@yahoo.com, dinucomanescu@yahoo.com, ioan.filipoiu@upb.ro, cristian.alionte@upb.ro

This Publication has to be referred as: Comanescu, A[driana]; Comanescu $\mathrm{D}$ [inu]; Filipoiu I[oan] D[an] \& Alionte C[ristian] G[abriel] (2010). About Spatial Rigid Memory Characteristics, Chapter 51 in DAAAM International Scientific Book 2010, pp. 583-592, B. Katalinic (Ed.), Published by DAAAM International, ISBN 978-3901509-74-2, ISSN 1726-9687, Vienna, Austria

DOI: $10.2507 /$ daaam.scibook.2010.51 


\section{Introduction}

The rigid memories materialized by planar or spatial cams are the main kinematical elements of various mechanisms applied in industrial equipments (food processing industry, textile industry, manufacturing processes, etc.) and robotics (Antonescu, 2005; Ivănescu, 2007).

The problem of cam planar mechanisms synthesis is comprehensively presented in the literature (Bishop, 2002; Hernandez et al., 2002). This problem is the only approach in some particular cases concerning cylindrical or truncated conic cams (Bloch 2003). This method presume that the spatial cams profile parameters can be determined for various motion laws of a follower which is in contact with the lateral or frontal spatial cam surface (Fig.1). After the calculation of the parameters, by using parametric CAD software, can be design cylindrical, conical and hyperbolical cams. The analytical synthesis of the spatial cam profile, presented in this paper, connected to adequate CAD software is firstly presented in the specialty literature.
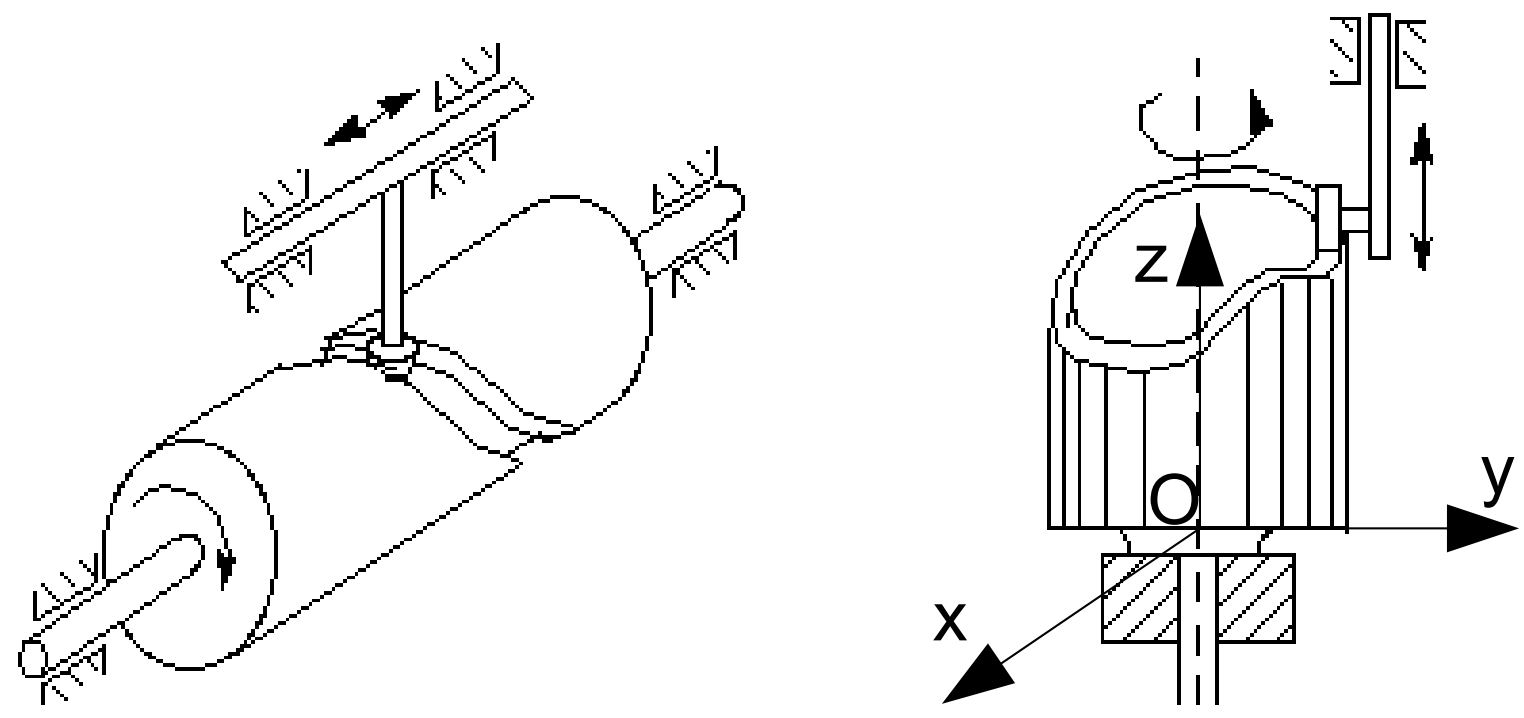

Fig. 1. Spatial cams

\section{Cylindrical cams synthesis}

In order to determine the line profile parameters of the cylindrical cam it is necessary to choose the following data: $\mathrm{T}$-cam rotation period, $\mathrm{r}$ - cam cylinder radius, $\mathrm{LV}=\mathrm{p}-$ follower displacement amplitude and motion law for the follower.

By developing the cam cylinder a rectangle is obtain, with the length $\mathrm{LH}=2 \pi \mathrm{r}$ and the height equal to $\mathrm{LV}$ to which a $\mathrm{xOy}$ reference system is attached. The current point of the profile line is $\mathrm{A}\left(\mathrm{x}_{\mathrm{AK}}, \mathrm{y}_{\mathrm{AK}}\right)$. In this reference system its coordinates depends on the law for the follower Table 1 and their 3D model representation being given in Fig. 2-4. 


\begin{tabular}{|c|c|}
\hline Law & Parametric equations in XOY reference system \\
\hline 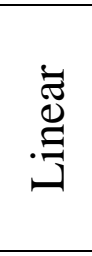 & $\begin{array}{l}\mathrm{x}_{\mathrm{AK}}=\mathrm{c} 1+\mathrm{k} 1 \cdot \mathrm{t}_{\mathrm{K}} \\
\mathrm{y}_{\mathrm{AK}}=\mathrm{if}\left[\mathrm{t}_{\mathrm{K}} \leq \frac{\mathrm{T}}{2}, \mathrm{c} 2+\mathrm{k} 2 \cdot \mathrm{t}_{\mathrm{K}}, \mathrm{LV}-\mathrm{y}_{\mathrm{A}}\left(\mathrm{t}_{\mathrm{K}-\frac{\mathrm{km}}{2}}\right)\right]\end{array}$ \\
\hline 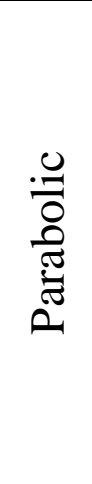 & $\begin{array}{rl}x_{A K}=k 1 \cdot t_{K} & r\left[t_{\mathrm{AK}}=\frac{T}{4},\left(\frac{2 \cdot p}{T^{2}} \cdot t_{k}^{2}\right), \text { if }\left[\left(\frac{T}{4} \leq t_{K} \leq \frac{3 \cdot T}{4}\right), \frac{-8}{T^{2}} \cdot p\right.\right. \\
\cdot & t_{k}^{2}+\left(\frac{8}{T^{2}} \cdot p \cdot t_{k}^{2}\right)-p, \frac{8}{T^{2}} \cdot p \cdot t_{k}^{2}-16 \cdot \frac{p}{T} \cdot t_{k} \\
& +8 \cdot p]]\end{array}$ \\
\hline 壳 & $\begin{aligned} \mathrm{x}_{\mathrm{AK}}=\mathrm{k} 1 \cdot \mathrm{t}_{\mathrm{K}} & \\
\mathrm{y}_{\mathrm{AK}}=\text { if }\left[\mathrm{t}_{\mathrm{K}}\right. & \leq \frac{\mathrm{T}}{2},\left(\frac{2 \cdot \mathrm{p}}{\mathrm{T}^{2}} \cdot \mathrm{t}_{\mathrm{k}}-\frac{\mathrm{p}}{2 \cdot \pi} \cdot \sin \left(4 \cdot \frac{\pi}{\mathrm{T}} \cdot \mathrm{t}_{\mathrm{k}}\right)\right), 2 \cdot \mathrm{p} \\
& \left.-2 \cdot \frac{\mathrm{p}}{\mathrm{T}} \cdot \mathrm{t}_{\mathrm{k}}+\frac{\mathrm{p}}{2 \cdot \pi} \sin \left(4 \cdot \frac{\pi}{\mathrm{T}} \cdot \mathrm{t}_{\mathrm{k}}\right)\right]\end{aligned}$ \\
\hline
\end{tabular}

Tab. 1. The coordinates of the profile current point

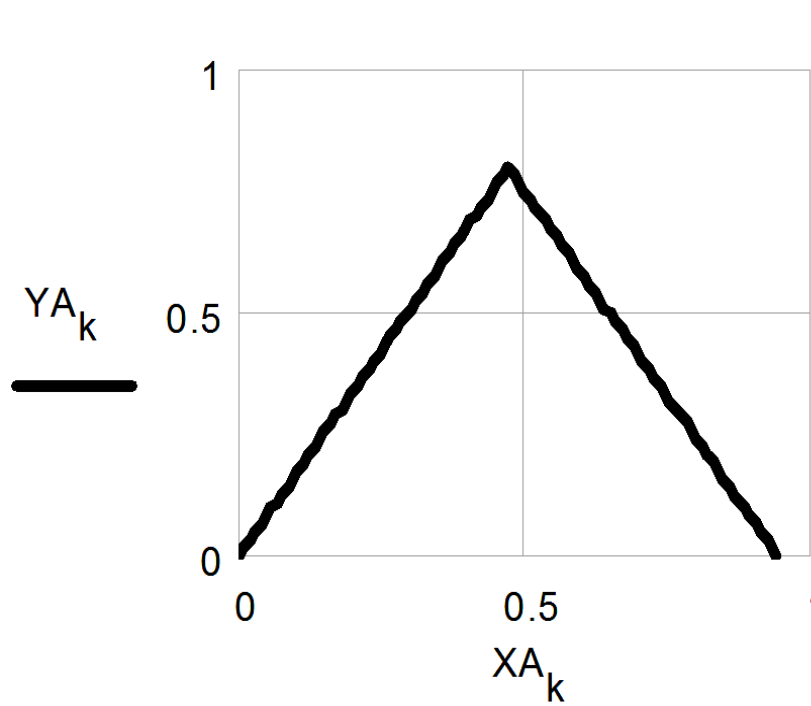

a)

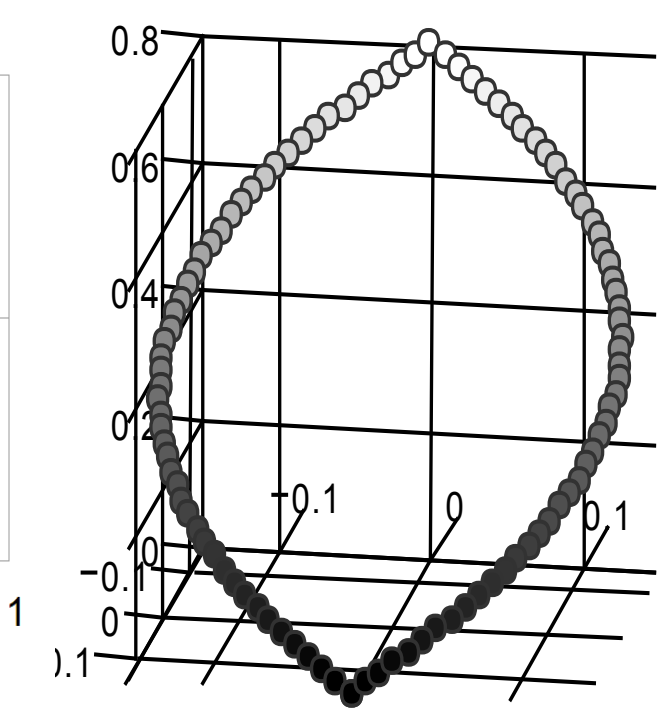

b)

Fig. 2. 3D graphic model representation of linear cam: a) Curve representation; b) Spatial curve profile line 
Comanescu, A.; Comanescu, D.; Filipoiu, I.D. \& Alionte, C.G.: About ...

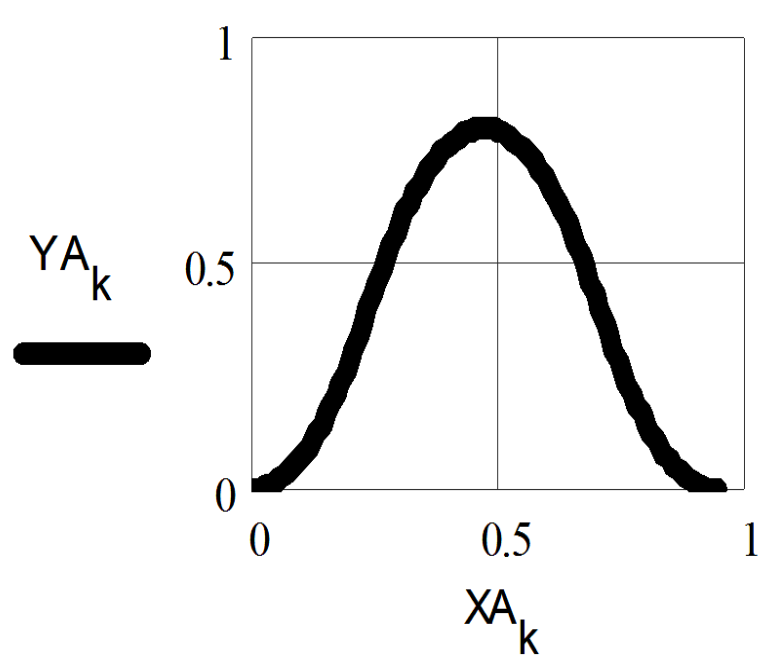

a)

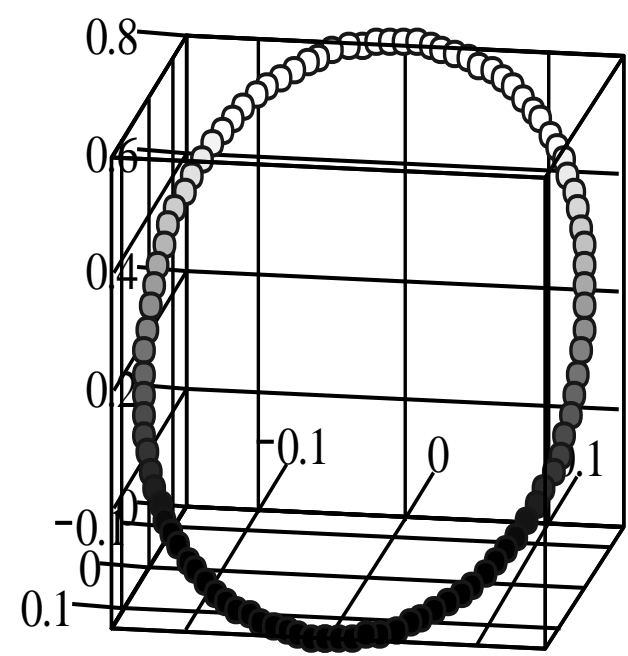

b)

Fig. 3. 3D graphic model representation of parabolic cam: a) Curve representation; b) Spatial curve profile line

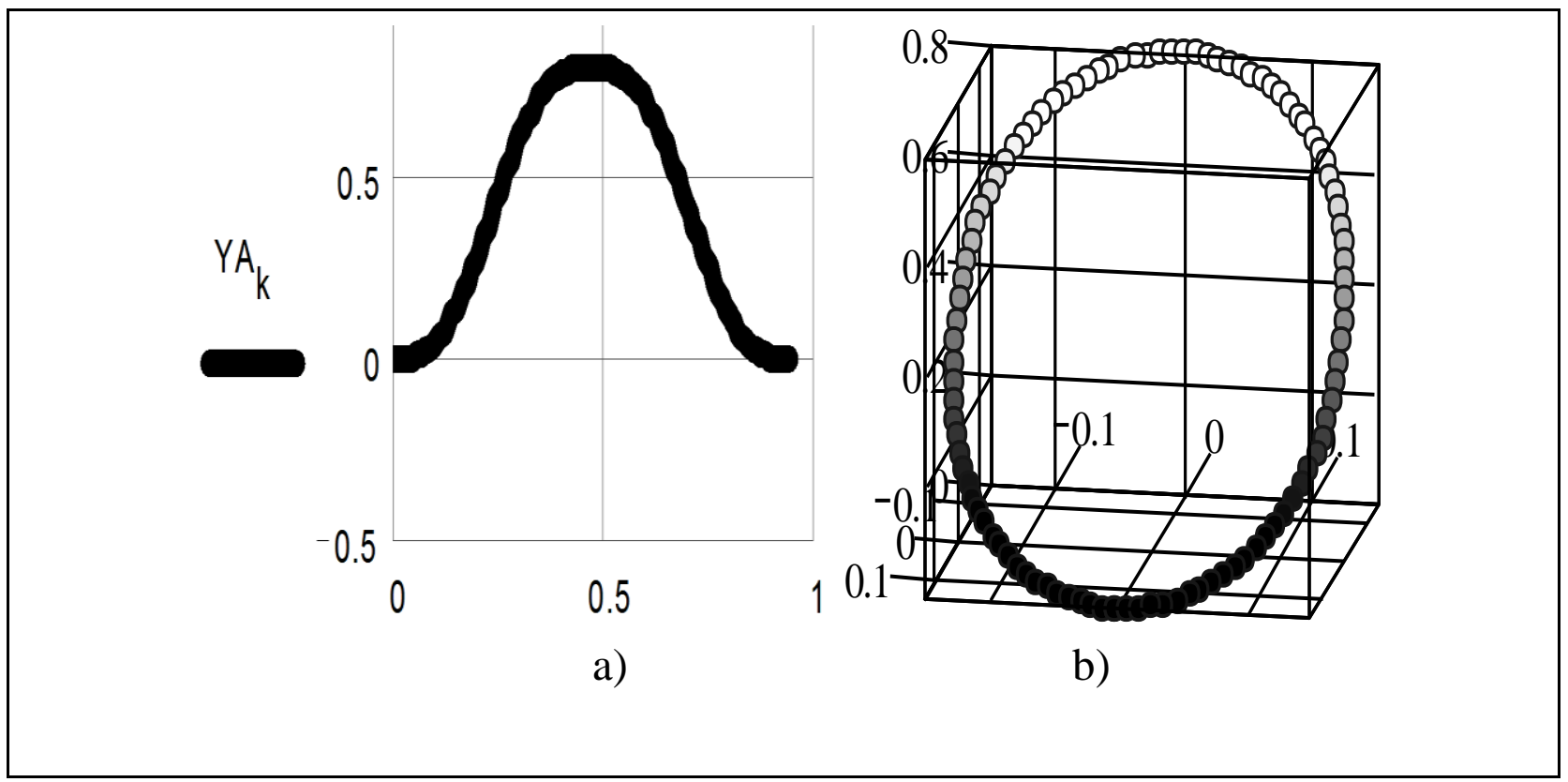

Fig. 4. 3D graphic model representation of parabolic cam: a) Curve representation; b) Spatial curve profile line

The spatial curve has the following parameter equations

$$
\begin{aligned}
& \mathrm{x}_{\mathrm{k}}=\mathrm{r} \cos \left(\psi_{\mathrm{k}}\right) \\
& \mathrm{y}_{\mathrm{k}}=\mathrm{r} \sin \left(\psi_{\mathrm{k}}\right)
\end{aligned}
$$




$$
\mathrm{z}_{\mathrm{Ak}}=\mathrm{y}_{\mathrm{Ak}}
$$

where $\psi_{k}=\frac{X_{A k}}{r}$.

The cylindrical cams model may be achieved by using adequate design software such as AutoCAD, Adams or SolidWorks. The parameters of the spatial curves profile are calculated and are given in Table 3. By applying a parameterization design, using CAD software, the design of such cams can be obtain, which are presented in Fig. 5.

\begin{tabular}{|l|l|l|l|l|l|l|l|l|l|}
\hline & \multicolumn{3}{|c|}{ Linear motion law } & \multicolumn{3}{c|}{ Parabolic motion law } & \multicolumn{3}{c|}{ Sinus motion law } \\
\hline $\mathrm{k}$ & $\mathrm{x}$ & $\mathrm{y}$ & $\mathrm{Z}$ & $\mathrm{x}$ & $\mathrm{y}$ & $\mathrm{Z}$ & $\mathrm{x}$ & $\mathrm{y}$ & $\mathrm{Z}$ \\
\hline 0 & 0.15 & 0 & 0 & 0.15 & 0 & 0 & 0.15 & 0 & 0 \\
\hline 10 & 0.121 & 0.088 & 0.16 & 0.121 & 0.088 & 0.064 & 0.121 & 0.088 & 0.039 \\
\hline 20 & 0.055 & 0.139 & 0.304 & 0.055 & 0.139 & 0.231 & 0.055 & 0.139 & 0.217 \\
\hline 30 & -0.037 & 0.145 & 0.464 & -0.037 & 0.145 & 0.518 & -0.037 & 0.145 & 0.525 \\
\hline 40 & -0.116 & 0.096 & 0.624 & -0.116 & 0.096 & 0.723 & -0.116 & 0.096 & 0.749 \\
\hline 50 & -0.15 & $9,42 \mathrm{E}+00$ & 0.784 & -0.15 & $9,42 \mathrm{E}+00$ & 0.799 & -0.15 & $9,42 \mathrm{E}+00$ & 0.8 \\
\hline 60 & -0.127 & -0.08 & 0.656 & -0.127 & -0.08 & 0.748 & -0.127 & -0.08 & 0.771 \\
\hline 70 & -0.055 & -0.139 & 0.496 & -0.055 & -0.139 & 0.569 & -0.055 & -0.139 & 0.583 \\
\hline 80 & 0.037 & -0.145 & 0.336 & 0.037 & -0.145 & 0.282 & 0.037 & -0.145 & 0.275 \\
\hline 90 & 0.116 & -0.096 & 0.176 & 0.116 & -0.096 & 0.077 & 0.116 & -0.096 & 0.051 \\
\hline 100 & 0.15 & $-9,42 \mathrm{E}+00$ & 0.016 & 0.15 & $-9,42 \mathrm{E}+00$ & $6.4 \mathrm{E}-4$ & 0.15 & $-9,42 \mathrm{E}+00$ & $4,21 \mathrm{E}-02$ \\
\hline
\end{tabular}

Tab. 2. The profile spatial coordinates for cylindrical cams

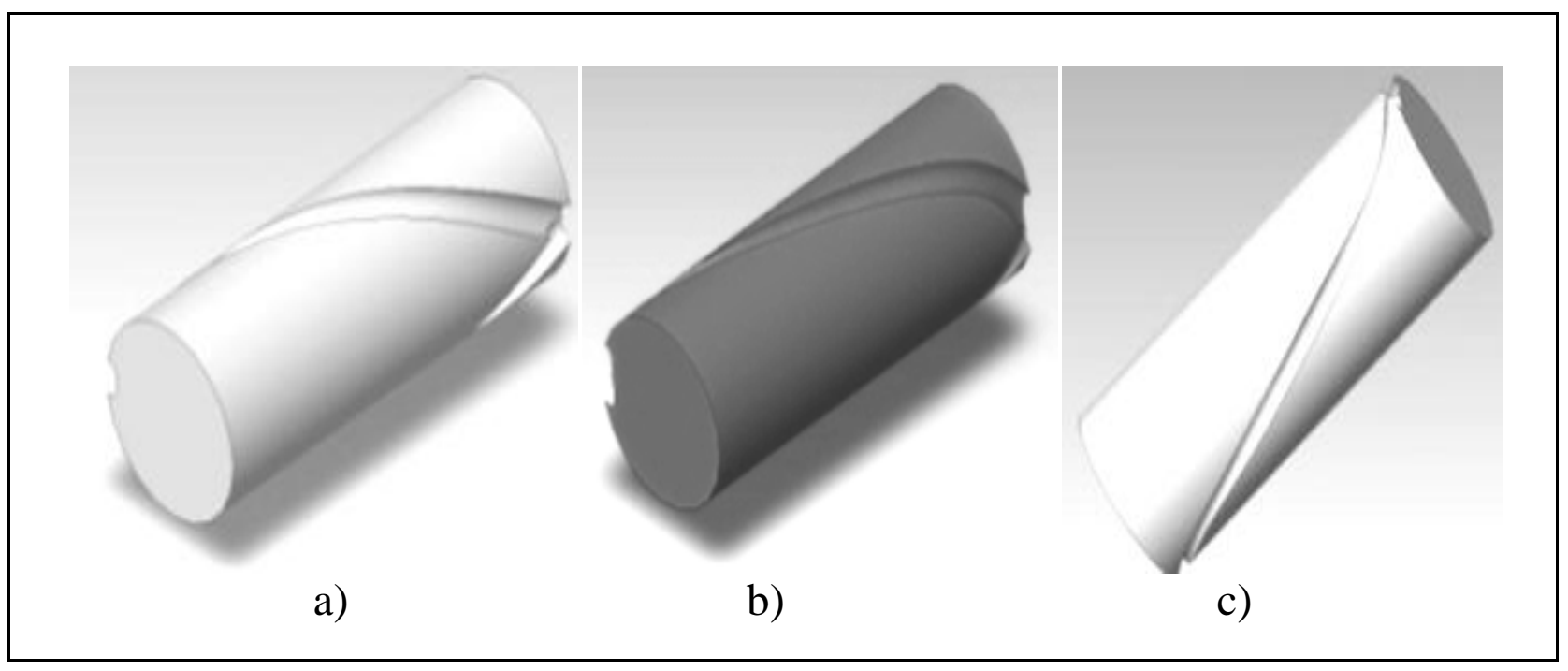

Fig. 5. Cylindrical cam: a) linear; b) parabolic; c) sin cylindrical

\section{Truncated conic cams synthesis}

The follower motion law on the $\mathrm{Oz}$ axis is imposed by the technological process. The characteristics to the truncated cone are presented in Fig.6

The truncated cone has the bases radius respectively $\mathrm{R}$ and $\mathrm{r}$. The basis circle length is denoted by LH. The follower motion amplitude on $\mathrm{Oz}$ is LV. The current 
point coordinates of the curve placed on the truncated conical surface, $A\left(x, y, z_{A}\right)$ may be deduced using the Fig. 6 and the following relations:

$$
\begin{gathered}
\mathrm{k}_{1}=\frac{\mathrm{LH}}{\mathrm{T}} \\
\mathrm{k}_{2}=2 \frac{\mathrm{LV}}{\mathrm{T}} \\
\chi=\operatorname{atan}\left(\frac{\mathrm{R}-\mathrm{r}}{\mathrm{LV}}\right) \\
\mathrm{H}=\frac{\mathrm{R}}{\tan \mathrm{L}(\mathrm{x})} \\
\mathrm{g}=\left(\mathrm{H}-\mathrm{ZA_{ \textrm {k } }}\right) \cdot \tan \chi \\
\mathrm{g}_{\mathrm{k}}=\sqrt{\left.\rho^{2}+(\mathrm{H}-\mathrm{ZA})_{\mathrm{k}}\right)^{2}} \\
\mathrm{G}=\sqrt{\mathrm{H}^{2}+\mathrm{R}^{2}} \\
\mathrm{p}=\frac{\xi}{\mathrm{T}} \\
\mathrm{U}_{\mathrm{k}}=\mathrm{p} \cdot \kappa_{\mathrm{k}} \\
\kappa_{\mathrm{k}}=\frac{s_{\mathrm{k}}}{\rho_{\mathrm{k}}}
\end{gathered}
$$

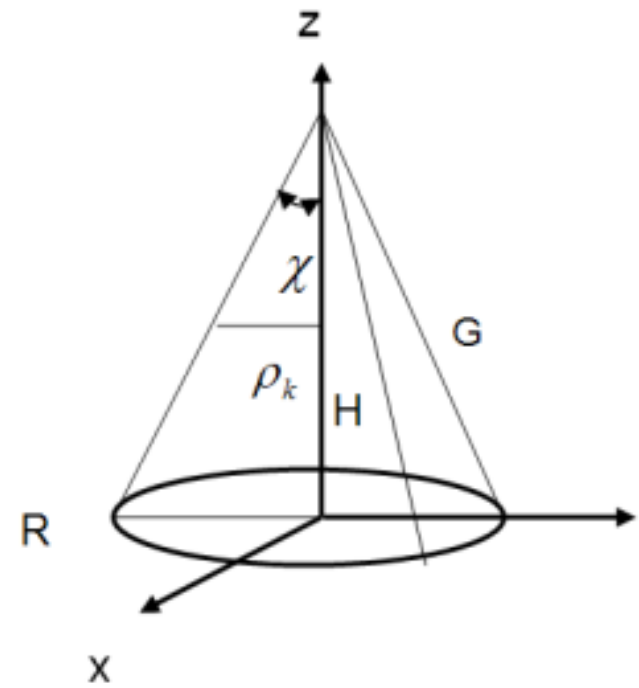

a)

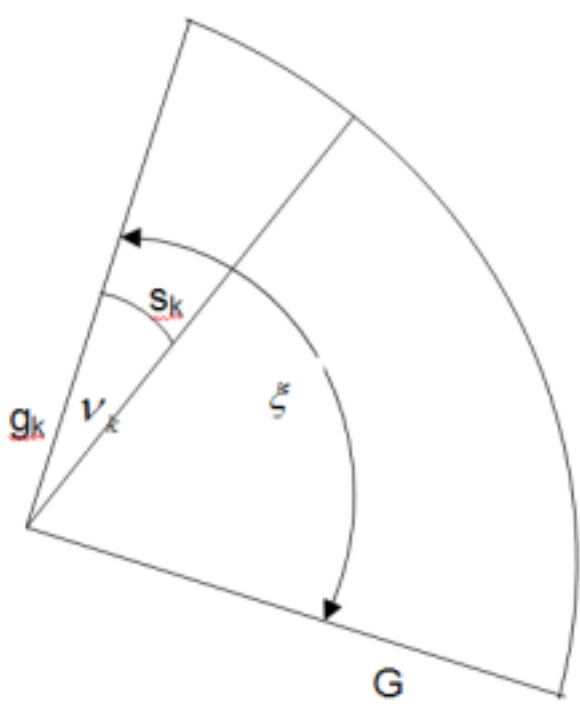

b)

Fig. 6. Characteristics to the truncated cone: a) The conic surface; b) the developed surface 
Thus

$$
\begin{aligned}
& x_{k}=\rho_{k} \cdot \cos \left(\kappa_{k}\right) \\
& y_{k}=\rho_{k} \cdot \sin \left(\kappa_{k}\right)
\end{aligned}
$$

In function of the adopted motion law of the follower $Z A_{k}$ (Tab.4) may be determined.

\begin{tabular}{|c|c|c|}
\hline Law & Parametric equations in XOY reference system \\
\hline Linear & $t_{k} \epsilon\left[0, \frac{T}{2}\right] \quad Z A_{k}=c 2+k 2 \cdot t_{k}$ \\
& $t_{k} \epsilon\left[\frac{T}{2}, T\right] \quad Z A_{k}=L V+Z A_{k-\frac{k m}{2}}$ \\
\hline \multirow{2}{*}{ Parabolic } & $t_{k} \epsilon\left[0, \frac{T}{4}\right] \quad Z A_{k}=\frac{8 \cdot p}{T^{2}} \cdot t_{k}^{2}$ \\
& $t_{k} \epsilon\left[\frac{T}{4}, \frac{3 \cdot T}{4}\right] \quad Z A_{k}=\frac{-8 \cdot p}{T^{2}} \cdot t_{k}^{2}+\frac{8 \cdot p}{T} \cdot t_{k}-p$ \\
\hline Sinus & $t_{k} \epsilon\left[\frac{3 \cdot T}{4}, T\right] \quad Z A_{k}=\frac{-8 \cdot p}{T^{2}} \cdot t_{k}^{2}+\frac{16 \cdot p}{T} \cdot t_{k}+8 p$ \\
& $t_{k} \epsilon\left[0, \frac{T}{2}\right] \quad Z A_{k}=\frac{16 \cdot p}{T} \cdot t_{k}-\frac{p}{2 \cdot \pi} \sin \left(\frac{4 \cdot \pi}{T} \cdot t_{k}\right)$ \\
& $t_{k} \epsilon\left[\frac{T}{2}, T\right] \quad Z A_{k}=2 \cdot p-\frac{2 \cdot p}{T} \cdot t_{k}+\frac{p}{2 \cdot \pi} \sin \left(\frac{4 \cdot \pi}{T} \cdot t_{k}\right)$ \\
\hline
\end{tabular}

Tab. 3. The motion law parameters

For example, by adopting the following values for $T=2 \mathrm{~s}, R=0,15 \mathrm{~m}$, $r=0.08 m, L V=0.8 m$ there are determined the coordinates of the spatial curve current point for different motion laws, Table 4.

\begin{tabular}{|c|c|c|c|c|c|c|c|c|c|}
\hline & \multicolumn{2}{|c|}{ Linear motion law } & \multicolumn{3}{c|}{ Parabolic motion law } & \multicolumn{2}{c|}{ Sinus motion law } \\
\hline $\mathrm{k}$ & $\mathrm{X}$ & $\mathrm{y}$ & $\mathrm{Z}$ & $\mathrm{X}$ & $\mathrm{y}$ & $\mathrm{Z}$ & $\mathrm{X}$ & $\mathrm{y}$ & $\mathrm{Z}$ \\
\hline 0 & 0.15 & 0 & 0 & 0.15 & 0 & 0 & 0.15 & 0 & 0 \\
\hline 10 & 0.11 & 0.08 & 0.16 & 0.117 & 0.085 & 0.064 & 0.119 & 0.086 & 0.039 \\
\hline 20 & 0.038 & 0.116 & 0.32 & 0.039 & 0.121 & 0.256 & 0.04 & 0.122 & 0.245 \\
\hline 30 & -0.033 & 0.103 & 0.48 & -0.032 & 0.097 & 0.544 & -0.031 & 0.096 & 0.555 \\
\hline 40 & -0.076 & 0.055 & 0.64 & -0.069 & 0.05 & 0.736 & -0.067 & 0.049 & 0.761 \\
\hline 50 & -0.08 & 0 & 0.8 & -0.08 & 0 & 0.8 & -0.08 & 0 & 0.8 \\
\hline 60 & -0.076 & -0.055 & 0.64 & -0.069 & -0.05 & 0.736 & -0.067 & -0.049 & 0.761 \\
\hline 70 & -0.033 & -0.103 & 0.48 & -0.032 & -0.097 & 0.544 & -0.031 & -0.096 & 0.555 \\
\hline 80 & 0.038 & -0.116 & 0.32 & 0.039 & -0.121 & 0.256 & 0.04 & -0.122 & 0.245 \\
\hline 90 & 0.11 & -0.08 & 0.16 & 0.117 & -0.085 & 0.064 & 0.119 & -0.086 & 0.039 \\
\hline 100 & 0.15 & 0 & 0 & 0.15 & 0 & 0 & 0.15 & 0 & 0 \\
\hline
\end{tabular}

Tab. 4. The profile spatial coordinates for truncated conical cams 
Comanescu, A.; Comanescu, D.; Filipoiu, I.D. \& Alionte, C.G.: About ...

These values were used in the modeling process using CAD software and the models are presented in Fig.7.

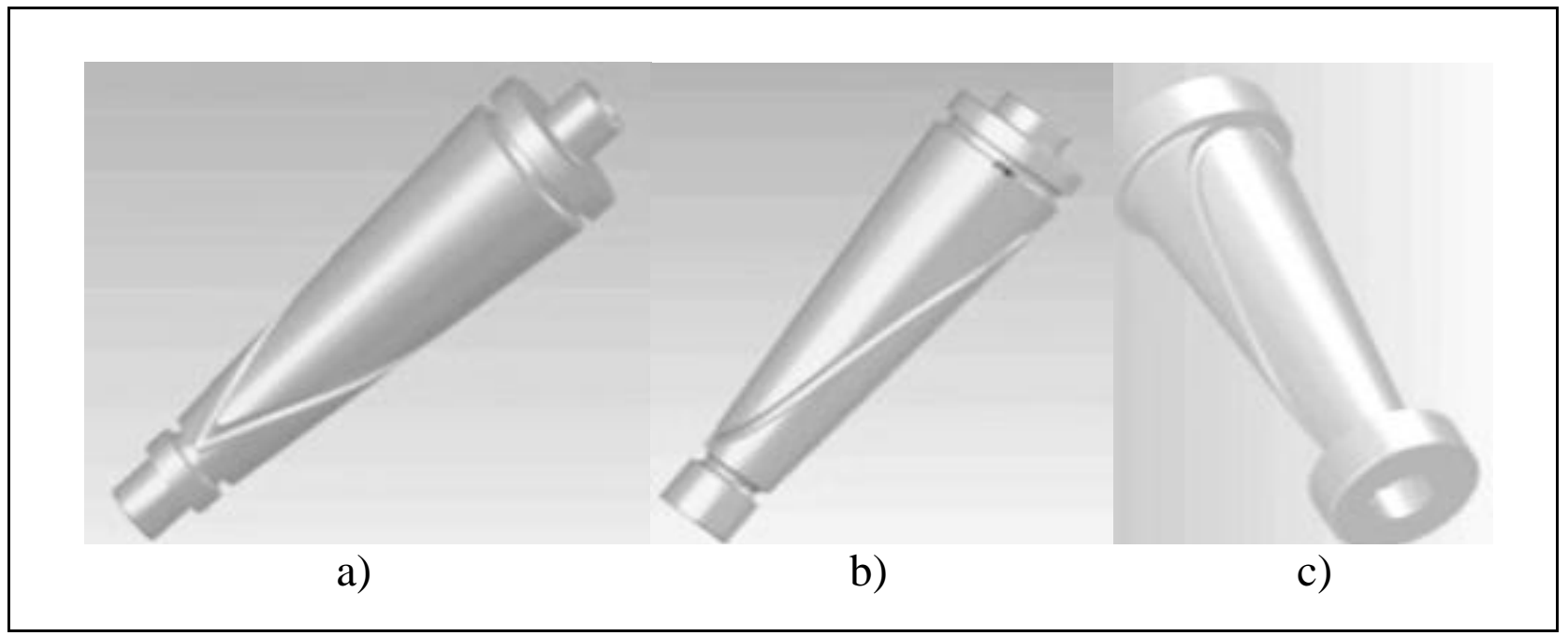

Fig. 7. Truncated conic cam: a) linear cam; b) parabolic cam; c) sin cam

\section{Hyperboloid cams synthesis}

In the case of the hyperboloid cams the follower extremity is in contact with the curve described on a hyperboloid.

Fig. 8. The hyperboloid

The hyperboloid surface (Fig.8) may be obtained by rotating a hyperbole around the $\mathrm{Oz}$ axis.

The hyperbole equation in the $\mathrm{yOz}$ reference system is

$$
\frac{\mathrm{Y}^{2}}{\mathrm{a}^{2}}-\frac{\mathrm{Z}^{2}}{\mathrm{~b}^{2}}=1
$$


where $b^{2}=c^{2}-a^{2}$ and $\mathrm{c}>a$.

The current point of the spatial curve placed on the hyperboloid is $A\left(x_{k}, y_{k}, z_{A k}\right) . z_{A k}$ is determined in function of the selected motion law, Table 5. The period of a complete rotation is $T$. $R$ and $r$ are respectively the maximum radius and the minimum one for the hyperboloid and $L V=p$ is the displacement amplitude on the Oz. For $k m$ time equal intervals can be determine $t_{k}=\frac{k T}{k m}$, where $k \epsilon[0, k m]$. At a $t_{k}$ time, the rotation angle is $\theta_{k}=t_{k} \cdot \omega$ (the cam angular velocity $\omega=\frac{2 \pi}{T}$ ).

The $\rho_{k}$ current radius is given by:

$$
\rho_{\mathrm{k}}=\sqrt{\mathrm{y}_{\mathrm{k}}^{2}}
$$

where $Y_{k}=\frac{a}{b} \sqrt{b^{2}+z_{A k}^{2}}$, thus

$$
\begin{aligned}
& x_{\mathrm{k}}=\rho_{\mathrm{k}} \cos \left(\theta_{\mathrm{k}}\right) \\
& \mathrm{y}_{\mathrm{k}}=\rho_{\mathrm{k}} \sin \left(\theta_{\mathrm{k}}\right)
\end{aligned}
$$

\begin{tabular}{|c|l|}
\hline Law & \multicolumn{1}{|c|}{ Parametric equations in XOY reference system } \\
\hline Linear & $\mathrm{t}_{\mathrm{k}} \in\left[0, \frac{\mathrm{T}}{2}\right] \quad \mathrm{ZA}_{\mathrm{k}}=\mathrm{c} 2+\mathrm{k} 2 \cdot \mathrm{t}_{\mathrm{k}}$ \\
& $\mathrm{t}_{\mathrm{k}} \in\left[\frac{\mathrm{T}}{2}, \mathrm{~T}\right] \quad \mathrm{ZA}_{\mathrm{k}}=-\mathrm{ZA}_{\mathrm{k}-\frac{\mathrm{km}}{2}}$ \\
\hline \multirow{3}{*}{ Parabolic } & $\mathrm{t}_{\mathrm{k}} \epsilon\left[0, \frac{\mathrm{T}}{4}\right] \quad \mathrm{ZA}_{\mathrm{k}}=\frac{8 \cdot \mathrm{p}}{\mathrm{T}^{2}} \cdot \mathrm{t}_{\mathrm{k}}{ }^{2}-\frac{\mathrm{p}}{2}$ \\
& $\mathrm{t}_{\mathrm{k}} \in\left[\frac{\mathrm{T}}{4}, \frac{3 \cdot \mathrm{T}}{4}\right] \quad \mathrm{ZA}_{\mathrm{k}}=\frac{-8 \cdot \mathrm{p}}{\mathrm{T}^{2}} \cdot \mathrm{t}_{\mathrm{k}}{ }^{2}+\frac{8 \cdot \mathrm{p}}{\mathrm{T}} \cdot \mathrm{t}_{\mathrm{k}}-\mathrm{p}+\frac{\mathrm{p}}{2}$ \\
\hline Sinus & $\mathrm{t}_{\mathrm{k}} \in\left[\frac{3 \cdot \mathrm{T}}{4}, \mathrm{~T}\right] \quad \mathrm{ZA}_{\mathrm{k}}=\frac{-8 \cdot \mathrm{p}}{\mathrm{T}^{2}} \cdot \mathrm{t}_{\mathrm{k}}{ }^{2}+\frac{16 \cdot \mathrm{p}}{\mathrm{T}} \cdot \mathrm{t}_{\mathrm{k}}+8 \mathrm{p}-\frac{\mathrm{p}}{2}$ \\
& $\mathrm{t}_{\mathrm{k}} \in\left[0, \frac{\mathrm{T}}{2}\right] \quad \mathrm{ZA}_{\mathrm{k}}=\frac{2 \cdot \mathrm{p}}{\mathrm{T}} \cdot \mathrm{t}_{\mathrm{k}}-\frac{\mathrm{p}}{2 \cdot \pi} \sin \left(\frac{4 \cdot \pi}{\mathrm{T}} \cdot \mathrm{t}_{\mathrm{k}}\right)-\frac{\mathrm{p}}{2}$ \\
$\mathrm{t}_{\mathrm{k}} \in\left[\frac{\mathrm{T}}{2}, \mathrm{~T}\right] \quad \mathrm{ZA} \mathrm{A}_{\mathrm{k}}=2 \cdot \mathrm{p}-\frac{2 \cdot \mathrm{p}}{\mathrm{T}} \cdot \mathrm{t}_{\mathrm{k}}+\frac{\mathrm{p}}{2 \cdot \pi} \sin \left(\frac{4 \cdot \pi}{\mathrm{T}} \cdot \mathrm{t}_{\mathrm{k}}\right)-\frac{\mathrm{p}}{2}$ \\
\hline
\end{tabular}

Tab. 5. The motion law parameters

By using the advanced design techniques were obtained the hyperboloid cams presented in Fig. 9. 


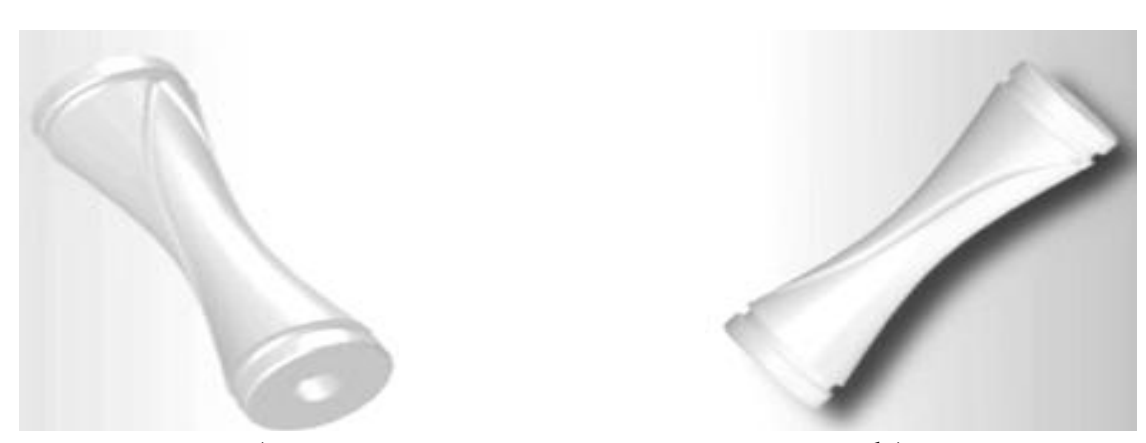

a)

b)

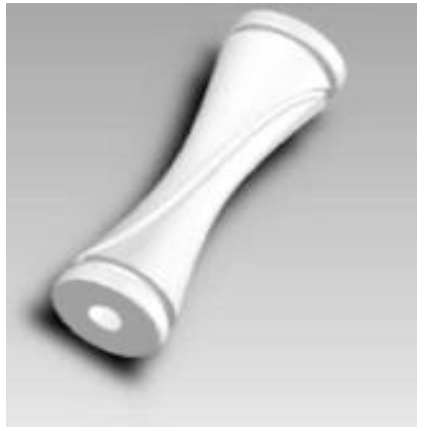

c)

Fig. 9. Hyperboloid cams: a) linear cam; b) parabolic cam; c) sin cam

\section{Conclusions}

In this paper it is presented an adequate synthesis method used for rigid memories defined by spatial cams. Our approach consists of finding the spatial parameters of the profile line, which can be determined by using calculus software. This motion lows provide CAD algorithm and are used to create a 3D model of the spatial cams. Of course they are other methods of cams synthesis but this one makes the manufacturing easier because this can be done using CAM software, which can is provided by CAD software.

The future researches include the 3D models database development to provide means or spatial cams synthesis based on follower motion laws.

\section{References}

Antonescu, P. (2005). Mechanism and Machine Science, PRINTECH. ISBN: 9737182916, Bucharest

Bishop, R.H. (2002). The mechatronics handbook, CRC Press. ISBN: 0849300665

Bloch, A.M. (2003). Nonholonomic mechanics and control, Springer-Verlag. ISBN: 0387955356, New York

Hernandez, A.; Pinto, C.; Petuya, V. \& Agirrebeitia, J. ( 2002). Mechanism theory, Serv. Publicaciones de la E. T. S. Ingenieros de Bilbao, ISBN: 8495809117, Bilbao

Ivănescu, M. (2007). From classical to modern mechanical engineering fundamentals, Edit. Acad. Romane, ISBN: 9789732715611, Bucharest 